\title{
LOW ALTITUDE AIRSHIPS FOR SEAMLESS MOBILE COMMUNICATION IN AIR TRAVEL
}

\author{
Madhu D ${ }^{1}$, Santhoshkumar M K ${ }^{1}$, Swarnalatha Srinivas ${ }^{2}$ and \\ Narendra Kumar $\mathrm{G}^{1}$ \\ ${ }^{1}$ Bangalore University, Bangalore. \\ ${ }^{2}$ Visvesvaraya Technological University, Belgaum. \\ madhudmnmegmail.com, santhoshkumarmksss@gmail.com, \\ gnarenk @yahoo.com \\ swarnalatha.ss@gmail.com
}

\begin{abstract}
The Aviation Administration policy prohibits the use of mobile phones in Aircraft during transition for the reason it may harm their communication system due to Electromagnetic interference. In case the user wants to access cellular network at higher altitudes, base station access is a problem. Large number of channels are allocated to a single user moving at high speed by various Base Stations in the vicinity to service the request requiring more resources. Low Altitude Platforms (LAPs) are provided in the form of Base stations in the Airships with antennas projected upwards which has direct link with the Ground Station. LAPs using LongEndurance Multi-Intelligence Vehicle (LEMVs) equipped with an engine for mobility and stable positioning against rough winds are utilized. This paper proposes a system that allows the passengers to use their mobiles in Aircraft using LAPs as an intermediate system between Aircraft and Ground station. As the Aircraft is dynamic, it has to change its link frequently with the Airships, MANETs using AODV protocol is established in the prototype using NS2 to provide the service and the results are encouraging.
\end{abstract}

\section{KEYWORDS}

Airships, AODV, Seamless Mobile Communication.

\section{INTRODUCTION}

Mobile phone is one of the active radio transmitter emitting electromagnetic radiations causing interference in the communication between the pilot and air traffic control unit [1]. The radiations also interfere with sensitive galvanometer based displays of older planes. The interference is caused because the cellular towers might be miles below the aircraft and the phone might have to transmit at its maximum power to establish a connection, increasing the risk of interference with Aircraft system. This paper proposes a system that operates at a frequency other than the Aircrafts communication system. Hence, the mobile can easily establish a connection with the Ground Station without having to transmit at its maximum power using Airships as the intermediate Base Stations. Airships are placed at appropriate positions along the path of the Aircraft in the required region, to provide communication services.

David C. Wyld et al. (Eds) : SAI, CDKP, ICAITA, NeCoM, SEAS, CMCA, ASUC, Signal - 2014 pp. 197-206, 2014. (C) CS \& IT-CSCP 2014

DOI : $10.5121 /$ csit.2014.41119 


\section{EXISTING METHOD}

GSMOB (GSM On Board) [2], [3] , [4] mobile services will allow airline passengers to use their own mobile terminals during certain stages of flight. Passengers are able to make and receive calls, send and receive SMS text messages and use GPRS functionality. The frequencies used for onboard communication are in the GSM1800 band. The main reasons for the selection of these bands is due to the small transmission power for an individual terminal when compared to the $900 \mathrm{MHz}$ band and emissions at higher frequencies produce higher path loss. A functional overview of a GSMOB system, Fig. 1.

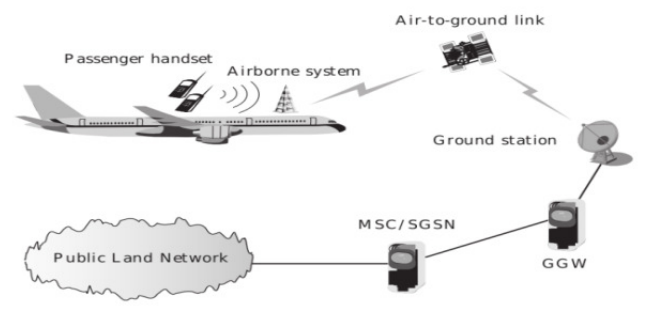

Figure 1. An overview of GSMOB system.

The challenges faced by the GSMOB system is to control the radio emissions of the mobile phones used by passengers, called Aircraft Mobile Stations (AcMS) and the on-board transmitters. AcMS try to connect to the cellular station even when the Aircraft is at cruising altitudes. Hence the AcMS transmit at higher power levels increasing the risk of interference. The log-on procedure used by all mobile phones on the market today is depicted in Fig. 2.

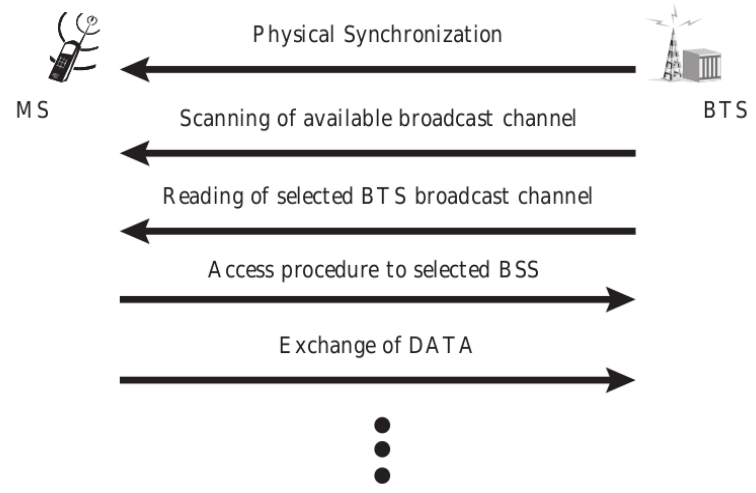

Figure 2. Mobile phones log-on procedure.

A technical approach for controlling the radio emissions aboard the aircraft is by making use of Network Control Unit (NCU) which prevents AcMS from attaching to the cellular network by injecting wideband noise of low power density into the relevant frequency bands, by which signals from cellular networks are effectively screened. Hence the cellular networks become invisible to the AcMS and they can transmit in a controlled manner by connecting to the Aircraft Base Transceiver Station (AcBTS) with the end to end architecture of the GSMOB, Fig 3. 


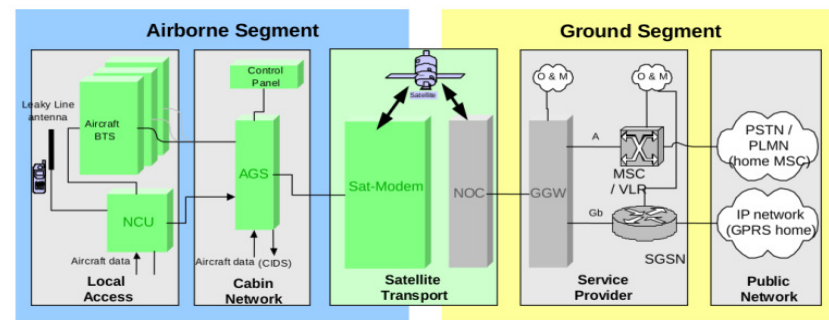

Figure 3. End to end architecture of GSMOB system.

Ground Segment- The ground segment consists of Service Provider Domain which includes Ground Gateway (GGW) and network components such as Mobile Switching Centre (MSC), Visitor Location Register (VLR) and Serving GPRS Support Node (SGSN) etc. The routing of the Aircraft traffic towards terrestrial backbone network of the Public Domain, Billing functions, mobility management are taken care of by the Service Provider Domain. The Public Network Domain of the Ground Segment provides the interconnection of the call, data or signalling communication to the relevant public network end points.

Airborne Segment- The Airborne Segment consists of the Local Access Domain and the Cabin Network Domain. The Local Access Domain contains the AcBTS providing GSM access for passengers AcMS and the NCU. The Cabin Network Domain contains the control panel and an Aircraft GSM Server (AGS). The control panel enables the crew to control the states of the GSMOB system. The AGS combines the GSM software on-board and interconnects the mobile phone system with the satellite modem.

\section{PROPOSED MODEL}

As the Aircraft enters the cruising altitude it spends much of its time during the flight in the range of 25,000 to 40,000 feet. In order to provide communication between the Aircraft and Ground station an Airship is used which is located at a height of about 20,000 feet. A scenario in which communication services are rendered to multiple Aircrafts which are separated by different Horizontal (minimum of 300m) and Vertical (minimum of 9.26km) distances [5] in region of interest is depicted, Fig. 4.

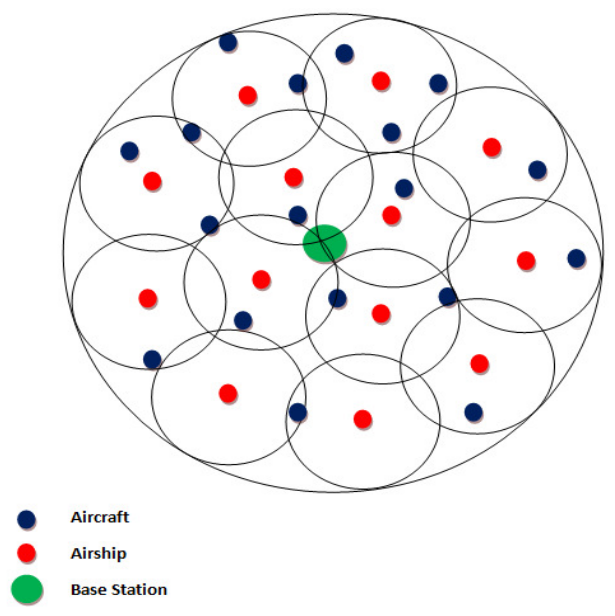

Figure 4. Airship assisted communication system for Aircraft. 


\subsection{Aircraft Communication System}

This system consists of dedicated transceivers for users in Aircraft to communicate with the Ground station. The Passengers can use their own terminals to make and receive calls, send and receive SMS and GPRS functionalities.

\subsection{Communication Channel}

Airships are located at lower altitudes (around 20,000 feet) compared to satellite, the effects such as signal delay, noise and interference are less. LEMV [6], [7] are large helium-filled balloon like

Airships with an aerodynamic "cigar" shape, about $91 \mathrm{~m}$ in length, $34 \mathrm{~m}$ in width, $26 \mathrm{~m}$ in Height, 38,000 cubic metres of envelope and has 4 x 350HP, 4 litre supercharged V8 diesel engines which can carry payloads of up to $2,750 \mathrm{lbs}$. The altitude of 20,000 feet is high enough to give local coverage of about $30 \mathrm{~km}$ in diameter and also offers the advantage of minimum wind speeds. LEMV can be optionally manned, remotely piloted or autonomously operated that consumes about 3,500 gallons of fuel to remain aloft continuously for a period of 21 days. The vehicle can fly at a loiter speed of 30kt and a dash speed of 80kt. There will be two types of antenna in airship: Master antenna and Slave antenna. The Airships along the path of the Aircraft are synchronised with one another with the help of the Master antenna to provide a regional coverage. The Master antennas of the Airships is also used to establish a continuous communication link with the ground station antenna. Slave antenna is used to capture the signal from the aircraft, the captured signal is then forwarded to the Master antenna and vice versa.

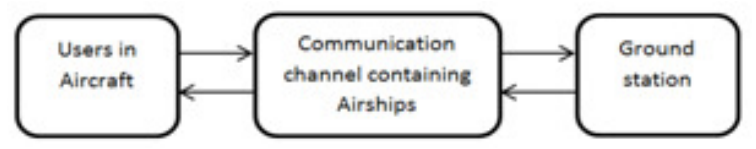

Figure 5. Proposed communication system.

\subsection{Ground Station}

It is the earth based communication station providing the communication link to the users in Aircraft. The earth station itself is usually an antenna that includes low noise amplifier, a down converter as well as an electronic receiver. The ground station is connected to Mobile Switching Centre for further switching operations.

\section{PROCESS}

In case a user in the Aircraft initiates a call, MANET routes the call to the Airship. The transceiver antenna in the Airship receives the call request and transmits it to the ground station which passes it to the MSC and connects the call to the desired user. Similarly in case a user on ground initiates the call is established and completed in the reverse direction. In case an Aircraft comes into the coverage region of particular Airship, slave antennae captures the call request signal and forwards it to the Master antenna which is in continuous communication with the ground station. In case the Aircraft comes out of the communication range of particular Airship, Handoff takes place. The process flow of the complete scenario is depicted, Fig. 6.

\section{ROUTING}

The Ad hoc On Demand Distance Vector (AODV) is a reactive routing Protocol, the routes are determined during requirement. AODV is capable of both unicast and multicast routing. It maintains the routes as long as they are needed by the sources. Each active node periodically broadcasts a Hello message that all its neighbours receive, in case a node fails to receive several Hello messages from a neighbour, a link break is detected. Data transmitted by source to an unknown destination broadcasts a Route Request (RREQ) for that destination. At each 
intermediate node a RREQ is received and a route to the source is created. In case the request receiving node is not the destination and does not have current route to the destination, it rebroadcasts the RREQ. In case the receiving node is the destination or has a current route to the destination, it generates a Route Reply (RREP). The RREP is unicast in a hop-by-hop fashion to the source. As the RREP propagates, each intermediate node creates and records a route to the destination. In case multiple RREPs are received by the source, the route with the shortest hop count is chosen and when a link break is detected during data flow, a Route Error (RERR) is sent to the source of the data in a hop-by-hop fashion, invalidates the route and reinitiates route discovery, Fig. 7.

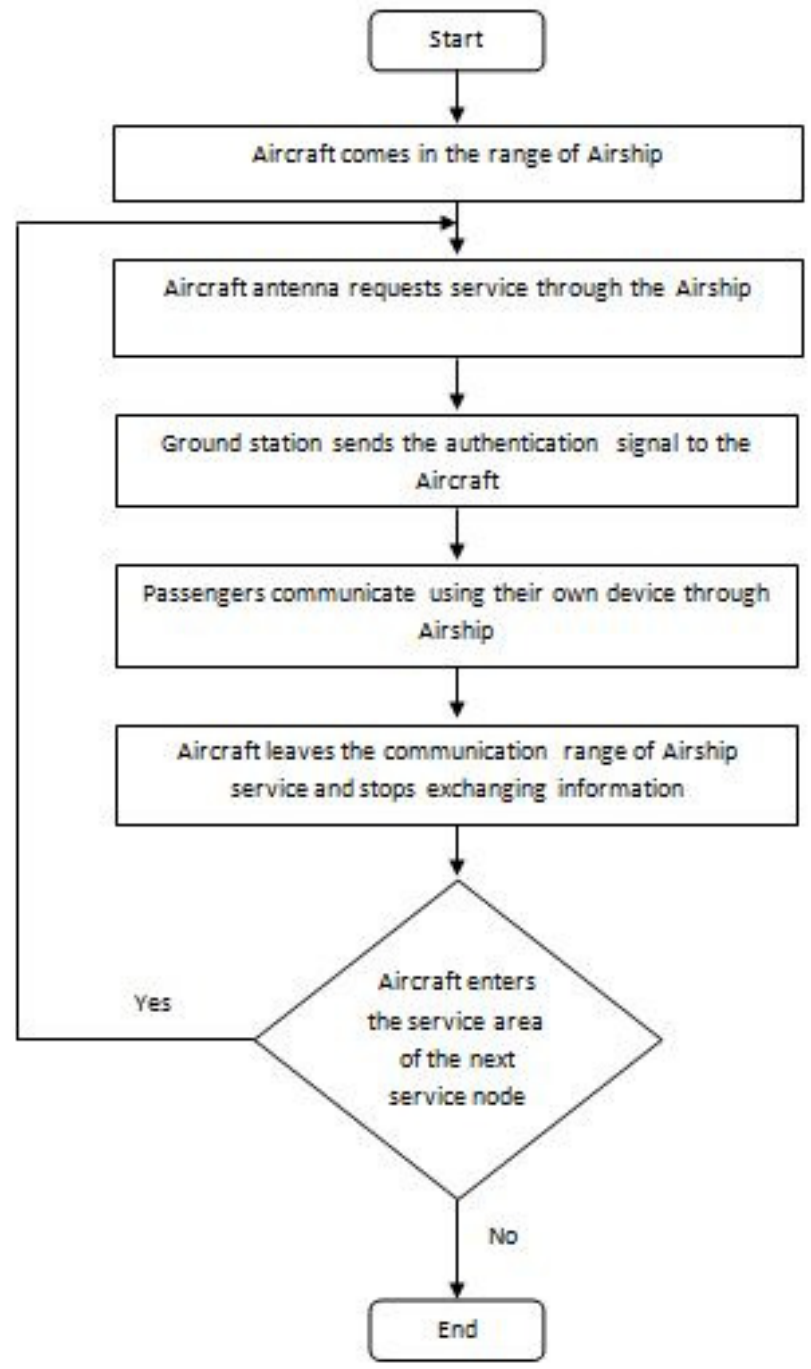

Figure 6. Process flow. 


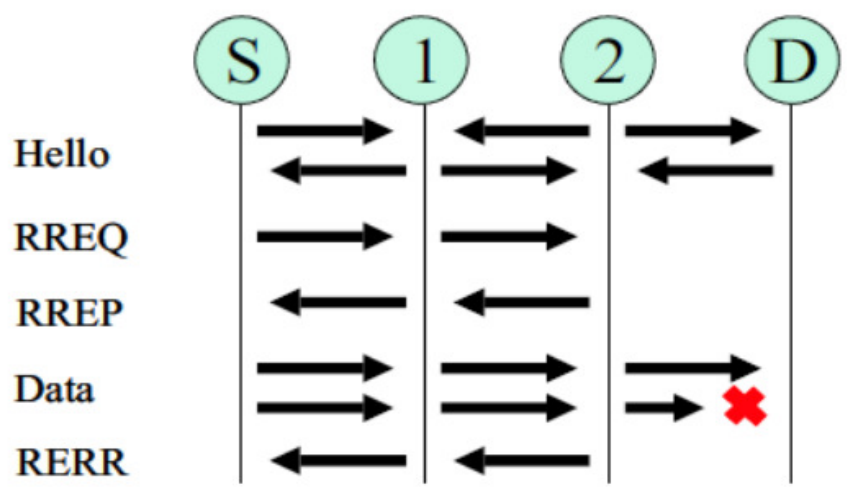

Figure 7. AODV Protocol Messaging.

\section{IMPLEMENTATION}

In order to provide communication, the Aircraft needs to consist of the subsystems, Fig. 8.

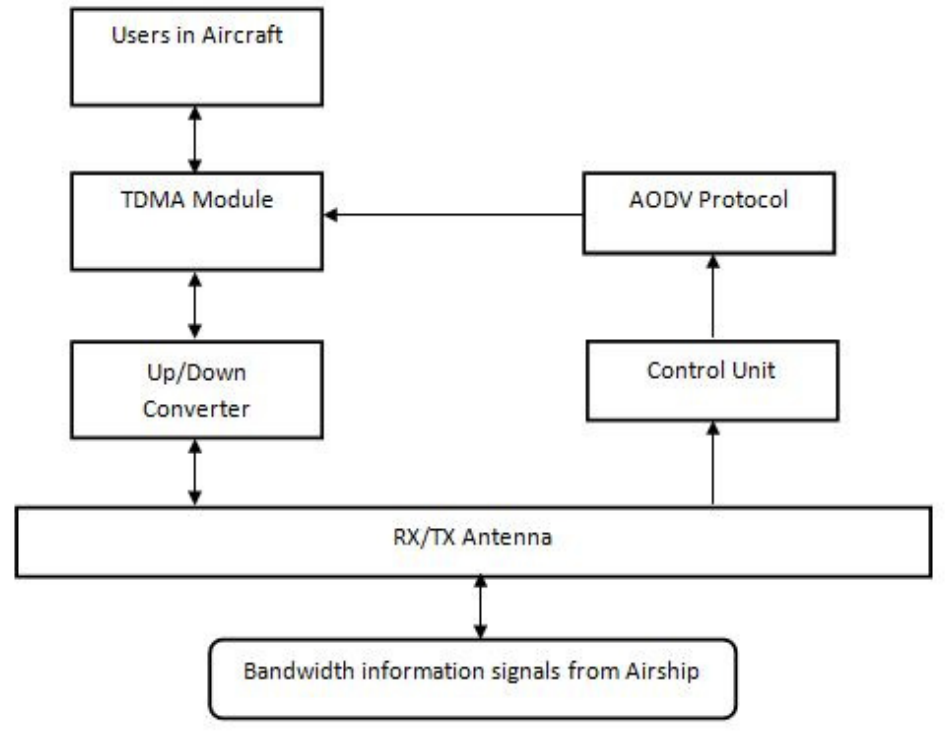

Figure 8. Aircraft system.

The users in the Aircraft requiring communication service can use their mobile devices which can be achieved by using a TDMA module aboard the Aircraft. The TDMA module allocates different time slots to different users to provide the service. The Up/Down converter in the Aircraft up converts the signal to be transmitted to antennas frequency and down converts the received signal to the mobiles operating frequency. The control unit present in the Aircraft receives the bandwidth information signals from the Airship and decides on the bandwidth to be allocated to the users in the Aircraft with the help of the TDMA module implementing AODV protocol to determine the shortest path and provide the point to point communication service to the users. 


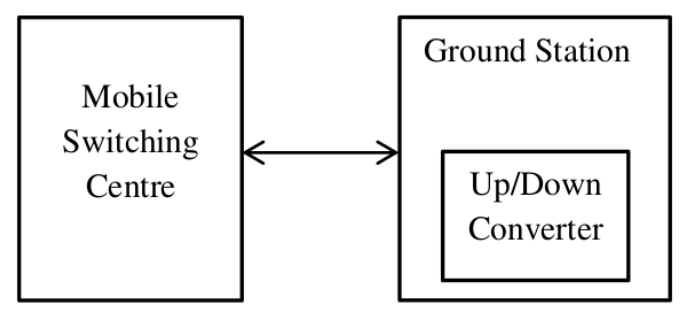

Figure 9. Ground station.

The Airship is the intermediate router between Aircraft and Ground station, also Guidance and Navigation system is used to control the position of the Airship. The Airships in a specified region have their own dedicated Ground station for transmitting and receiving the signal. The Ground stations antenna should be in line of sight with the closest Airships antenna and they should communicate continuously with each other to render the requested service. The ground station is synchronized with the MSC, Fig. 9 and the Up/Down converter in it has the same functionality as that of Aircraft's.

\section{SIMULATION AND RESULTS}

Basic Model Configuration: The nodes are positioned for Airships, Ground Station and Aircraft. Red colour nodes serve as Airships, Green nodes as Ground stations and Blue nodes as Aircrafts. Since an Airship provides circular coverage of $30 \mathrm{~km}$ diameter, they are placed consecutively along the path of Aircraft and all of these Airships are in sync with a dedicated Ground station depicted, Fig. 10.

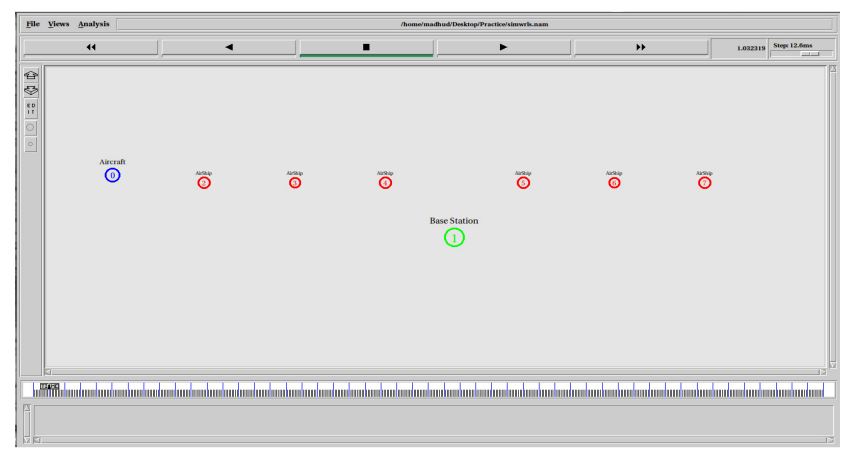

Figure 10. Basic Model of Simulation.

Experimental Analysis and Results: A TCL program is written to simulate the required topology of wireless network in NS2. The wireless simulation related parameters are defined as follows:

- Channel Type

- Radio-propagation model

- Network interface type

- MAC type

- Interface queue type

- Link layer type

- Antenna model

- Max packet in ifq

- Routing protocol
: Wireless

: $\quad$ Two Ray Ground

: Wireless Phy

: $\quad$ Mac/802.11

: $\quad$ Queue/ Drop Tail/ Pri Queue

: LL

: Antenna/Omni

: $\quad 50$

: $\quad$ AODV 


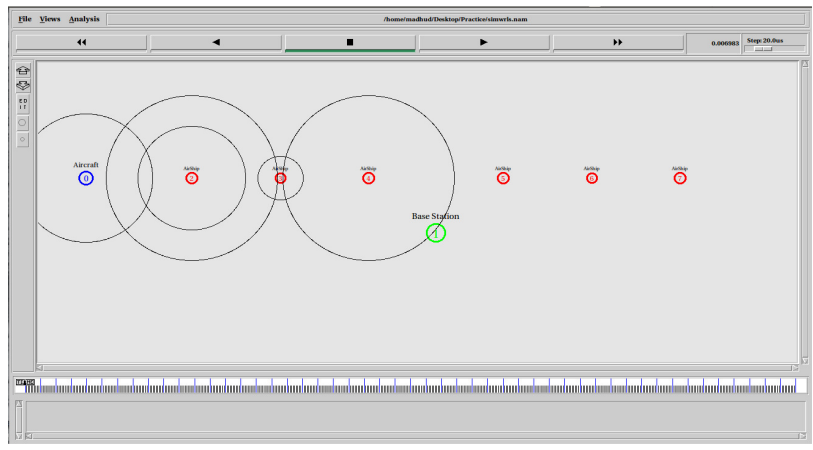

Figure 11. Aircraft linking with Airship.

In case an Aircraft comes within the communication range of an Airship, it sends request message to Ground station and in turn receives an authentication using Airships as an intermediate router. The exchange of information between Aircraft, Airship and the Ground station is depicted, Fig. 11. Gradually when the Aircraft comes out of the communication range of currently linked Airship, the Handoff to the next Airship takes place as depicted, Fig. 12.

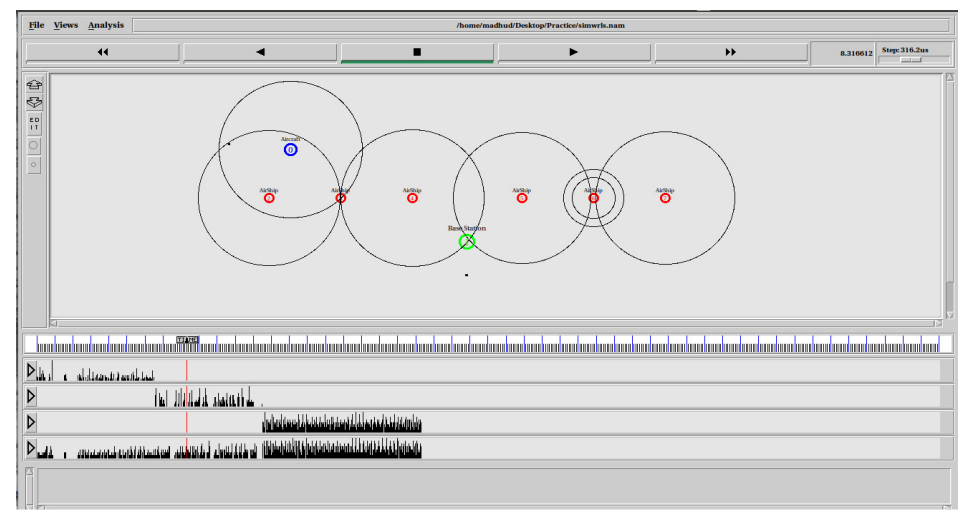

Figure 12. Aircraft changing its link to other Airship.

In case two aircrafts simultaneously arrive in a regional coverage area provided by dedicated Airships and Ground station, both the Aircrafts are provided with the communication services simultaneously, Fig. 13. The continuous communication services provided to passengers in multiple Aircrafts in different regions of interest is depicted, Fig. 14.

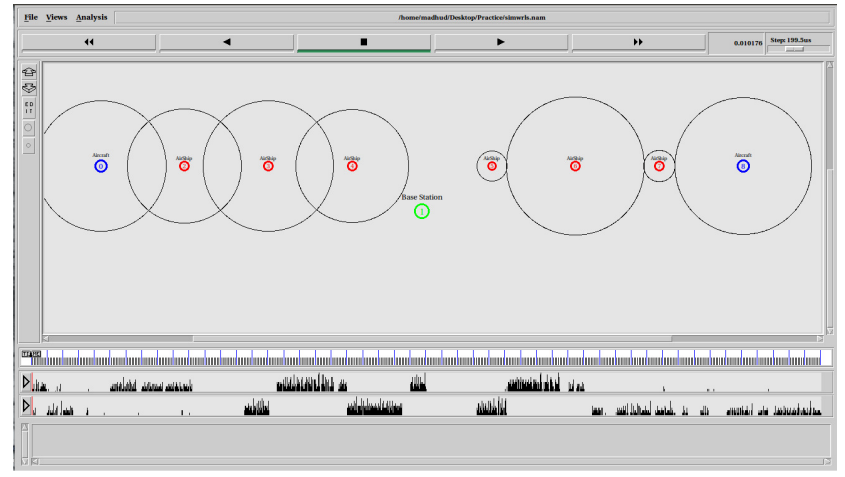

Figure 13. Two Aircrafts in a regional coverage of dedicated Airships and Ground station. 


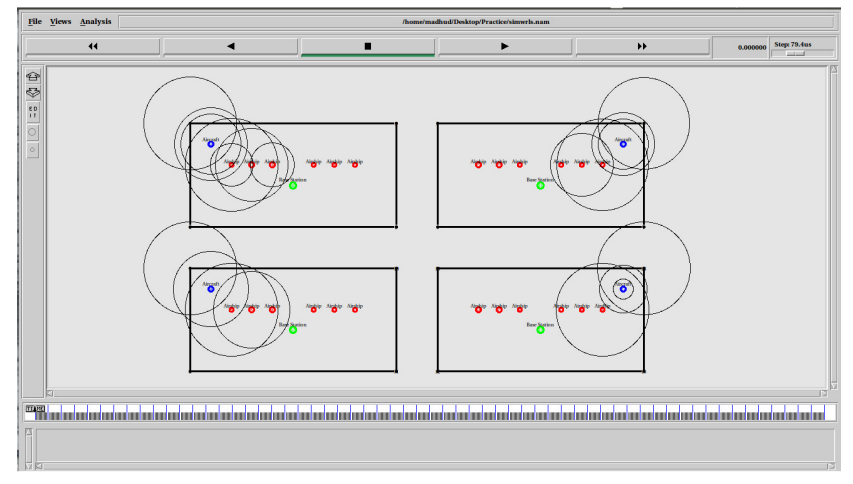

Figure 14. Continuous communication service in different regions of interest.

\section{CONCLUSION}

The paper aims to provide seamless communication services to the mobile users in the Aircraft, they are enabled to operate their own devices by using the communication system installed in the Aircraft. The Airship is used as an intermediate router to forward the information between Aircraft and ground station and vice-versa. In the proposed model the delay incurred by the signal along its path will be reduced. As communication services can be readily established in regions of interest, the proposed model can be utilized in relief measures for natural disasters like Floods, Earth quakes etc. Since there exists a line of sight between the Airships and the Ground station, the use of Airships to provide communication services to the users on ground is another possibility.

\section{REFERENCES}

[1] See Website-"http://en.wikipedia.org/wiki/Mobile_phones_on_aircraft".

[2] César Gutiérrez Miguélez, "GSM operation onboard aircraft", ETSI White Paper No. 4, January 2007.

[3] Carlos Gonzaga Lopez, "GSM ON BOARD AIRCRAFT", December 15, 2008.

[4] John Mettrop,"GSM On-Board Aircraft", Directorate of Airspace Policy Surveillance \& Spectrum Management.

[5] Aircraft Separation- "http://www.nokaviation.com/PPL_trg/rules.htm".

[6] See Website- "http://www.defenseindustrydaily.com/rise-of-the-blimpsthe-us-armys-lemv-06438/\#".

[7] See Website- "http://www.army-technology.com/projects/long-endurancemulti-intelligence-vehicle/".

[8] See Website- "http://www.slideshare.net/rishikeshims/aircraftcommunicationsystems"

[9] ETSI ETS 300 326-1, "Radio Equipment and Systems (RES)", "Terrestrial Flight Telephone System (TFTS)".

[10] ETSI ETS 300 326-2, "Radio Equipment and Systems (RES)", "Terrestrial Flight Telephone System (TFTS)".

[11] Network Simulator Tutorial- "http://www.isi.edu/nsnam/ns/tutorial".

[12] NS2 Range Calculation- "http://mailman.isi.edu/pipermail/nsusers/2012-August/072160.html".

[13] Data Transfer in NS2- "http://csis.bitspilani.ac.in/faculty/murali/resources/tutorials/ns2.htm". 


\section{AUTHORS}

Dr. Narendra Kumar G, born in Bangalore on 5th February, 1959. Obtained Masters Degree in Electrical Communication Engineering, (Computer Science \& Communication) from Indian Institute of Science, Bangalore, Karnataka, India in 1987. Was awarded $\mathrm{PhD}$ in Electrical Engineering(Computer Network) from Bangalore University, Bangalore, Karnataka, India in 2006.Currently Professor in the Department of Electronics \& Communication Engineering, University Visvesvaraya College of Engg., Bangalore University, Bangalore, held the positions of Associate Professor, Lecturer and Director of Students Welfare. Research interests include Mobile Communication, Wireless Communication, E-Commerce, Robotics and Computer Networks.

Dr. Swarnalatha Srinivas, born in Bangalore on 22nd October, 1964. Obtained Bachelors Degree in Electrical Engineering from University Visvesvaraya College of Engg., Bangalore, Karnataka, India in 1988. Obtained Masters degree in Power Systems, University Visvesvaraya College of Engg., Bangalore, Karnataka, India in 1992 and was awarded PhD under the guidance of Dr Narendra Kumar G in 2014. Currently Associate Professor in the Department of Electrical Engineering, Bangalore Institute of Technology, VTU, Bangalore.

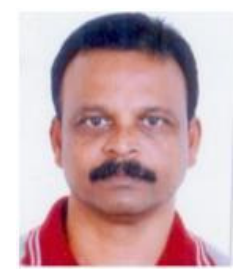

Madhu D and Santhoshkumar M K are research students under the guidance of Prof. Narendra Kumar G. 